\title{
Spacetime Averaged Null Energy Condition
}

\author{
Douglas Urban* and Ken D. Olum \\ Institute of Cosmology, Department of Physics and Astronomy, \\ Tufts University, Medford, MA 02155, USA.
}

\begin{abstract}
The averaged null energy condition has known violations for quantum fields in curved space, even when one considers only achronal geodesics. Many such examples involve rapid variation in the stress-energy tensor in the vicinity of the geodesic under consideration, giving rise to the possibility that averaging in additional dimensions would yield a principle universally obeyed by quantum fields. However, after discussing various procedures for additional averaging, including integrating over all dimensions of the manifold, we give here a class of examples that violate any such averaged condition.
\end{abstract}

PACS numbers: $04.62 .+\mathrm{v}$ 04.20.Gz

*Electronic address: Douglas.Urban@tufts.edu

$\dagger$ Electronic address: kdo@cosmos.phy.tufts.edu 


\section{INTRODUCTION}

General relativity itself places no restrictions on which geometries may be considered. If we want to exclude pathological phenomena, such as closed timelike curves, wormholes, or superluminal communication, we must appeal to restrictions on the stress-energy tensor of the sources, $T_{a b}$. For classical fields there is a variety of such energy conditions which all hold and have been used to exclude such behavior [1 14$]$.

The null energy condition (NEC) states that for a null vector $l^{a}$ we always have $T_{a b} l^{a} l^{b} \geq 0$. Other pointlike conditions often discussed, such as the weak, strong and dominant conditions, all imply the null energy condition. However, all pointlike conditions are violated by quantum fields. Even a simple vacuum plus two photon state possesses negative energy density in some regions.

We can instead consider the averaged null energy condition (ANEC),

$$
\int_{\gamma} d \lambda T_{a b} l^{a} l^{b} \geq 0
$$

where $\gamma$ is a null geodesic, $l^{a}$ the tangent to it, and $\lambda$ an affine parameter. Equation (1) can easily be violated with compactified dimensions, but we can restrict our attention to the case where $\gamma$ is an achronal geodesic, i.e., where no two points of $\gamma$ are also connected by a timelike path. Positive energy densities lead to gravitational lensing, which produces conjugate points, so in the absence of negative energy densities generic geodesics are chronal.

Requiring ANEC to hold only on achronal geodesics eliminates many known violations, while still ruling out many exotic phenomena [5]. ANEC always holds in Minkowski space [6, 7] (where all null geodesics are achronal), and along achronal geodesics surrounded by a flat tubular neighborhood [8] in curved space. Nevertheless, even "achronal ANEC" can be violated. One kind of violation found by Visser [9] can be produced by the scale anomaly for conformally coupled scalar fields. This effect is logarithmically suppressed and depends on the renormalization scale.

In addition, we recently found two ANEC violations that are present in conformally flat spacetimes (where Visser's anomaly vanishes) and do not depend on the renormalization scale [10]. Conformal transformations of conformally coupled fields are an easy case to analyze, as the transformation properties of the stress tensor are known [11]. If we write the conformally flat metric $\bar{g}_{a b}=\Omega^{2} \eta_{a b}$, the conformally coupled scalar field transforms as $\bar{\phi}=\Omega^{-1} \phi$, but the stress tensor does not transform simply. Instead, it has anomalous terms, so

$$
\bar{T}_{a b}=\Omega^{-2} T_{a b}+\text { curvature anomaly. }
$$

Our first construction utilizes a sequence of states in Minkowski space whose momentum is constrained to lie within a cone that lengthens and narrows as a parameter $\alpha \rightarrow 0$. As the limit is taken the states approach the vacuum, but the stress tensor near the origin grows more negative. Although the Minkowski space ANEC integral vanishes in the $\alpha \rightarrow 0$ limit, in a transformed space the integrand is weighted by $\Omega^{-4}$, which can enhance the negative contribution. For small enough $\alpha$ the curvature anomaly can be dwarfed by this negative contribution.

The second violation uses only the curvature anomaly, which can itself be negative.

One can consider transforming the ground state, so that the $\Omega^{-2} T_{a b}$ contribution to $\bar{T}_{a b}$ vanishes. It is worth noting that the transformation of the ground state is not in general 
the ground state in the new space. Using null coordinates defined in Minkowski space, with $u=(z-t) / \sqrt{2}$ and $v=(z+t) / \sqrt{2}$, the specific example illustrated in Ref. [10] is

$$
\Omega=\exp \left[\left(a+\frac{b x^{2}}{r^{2}}\right) e^{-\left(u^{2}+v^{2}+x^{2}+y^{2}\right) / r^{2}}\right]
$$

In both of these situations, the magnitude of the violation grows as the effect is more tightly constrained to the geodesic. This motivates us to inquire whether a version of ANEC that includes averaging in additional directions would avoid these violations. Indeed, if one's average includes timelike directions, one can use the null-contracted, timelike-averaged quantum energy inequality of Ref. [12] to limit the amount by which such an additionally averaged ANEC can be violated. In such a case, it is not possible to produce a sequence of states that give unlimited violation, as we did in Ref. [10] for ANEC alone.

For the curvature case, Eq. (3) gives the ANEC integral

$$
16 \sqrt{2 \pi} a(b-2 a) \beta / r^{3},
$$

where

$$
\beta=-\frac{1}{5760 \pi^{2}} .
$$

Since $\beta<0$, if we choose $b>2 a$, ANEC will be violated. In this particular case, additional averaging gives a positive result. Nevertheless, we show below that similar constructions can violate any generally averaged condition, and thus no additionally averaged version of ANEC is generally obeyed in curved spacetimes.

We work in units where $c=1$ and $\hbar=1$. Our sign conventions are $(+++)$ in the categorization of Misner, Thorne, and Wheeler [13].

\section{ADDITIONALLY AVERAGED NULL ENERGY CONDITIONS}

Averaging the null energy over a null geodesic eliminates many violations of the NEC, but even ANEC has violations. It might be that averaging in additional directions could eliminate these and yield a principle that all quantum fields would obey. But what do we mean by a more general average of NEC? If we establish a null vector field $l^{a}$ throughout spacetime, we can project the stress-energy tensor on this field and take the average,

$$
A_{4}=\int \sqrt{-g} d^{4} x T_{a b} l^{a} l^{b}
$$

but is not clear how we should define $l^{a}$.

In the case of the regular ANEC, we can start with a vector $l^{a}$ tangent to our null geodesic $\gamma$ at some initial point $p$. Such a vector is defined only up to rescaling, but such change (equivalent to a change of affine parameter) only affects the magnitude of the ANEC integral, not its sign. We then establish $l^{a}$ everywhere on the geodesic by parallel transport from $p$ to each destination point $x$.

We could attempt the same technique for averaging in more dimensions, but now there is more than one choice of path for the parallel transport. In general, when we work in curved space the resulting $l^{a}$ will depend on the path chosen. Flanagan and Wald [14] make the choice to transport $l^{a}$ along a geodesic from $p$ to $x$. This is well defined if one works inside a 
normal neighborhood. If one considers perturbations of flat space as done in Ref. [14], and as we will do below, one can transport $l^{a}$ in the unperturbed space-time without ambiguity. But in the general case, there may be no geodesic, or multiple geodesics, connecting $p$ and $x$, and the procedure does not work.

We can also consider averaging over more than a single geodesic but less than all the dimensions of the manifold. For example, let $\chi$ be a timelike line parametrized by proper time $\tau$. Start with a null vector $l^{a}$ at some point $p \in \chi$, and establish a null vector field $l^{a}$ on $\chi$ by parallel transport. Through each point of $\chi$ draw the null geodesic whose tangent vector is $l^{a}$. Then we can write

$$
A_{2}=\int d \tau d \lambda T_{a b} l^{a} l^{b}
$$

Similarly, we can average only over spacelike directions, but here we will encounter ambiguities. Given a spacelike 2 -surface $\Sigma$, let us establish a null vector field $l^{a}$ orthogonal to the surface at each point. These vectors generate a family of geodesics. We can take the integral over each one, to get

$$
A_{3}=\int_{\Sigma} \sqrt{g_{2}} d \sigma_{1} d \sigma_{2} \int T_{a b} l^{a} l^{b} d \lambda .
$$

Here $\sigma_{1}$ and $\sigma_{2}$ are the coordinates on the surface and $g_{2}$ the induced metric. The inner integral is to be taken over the geodesic generated by $l_{a}$ at each point.

The direction of $l^{a}$ is fixed by orthogonality, but we need to fix the magnitude. As before, we could try do to this via parallel transport, but that may depend on the path chosen. Thus this idea does not lead to a well-defined averaging procedure.

This process depends on the choice of the initial surface, even if the resulting null 3surface is fixed. Suppose we propagate our initial surface an affine distance $\lambda$ down each geodesic to get a new surface $\Sigma^{\prime}$. The geodesics may spread out or squeeze together between $\Sigma$ and $\Sigma^{\prime}$. Thus if we started with $\Sigma^{\prime}$ instead of $\Sigma$, we would have a different weighting of the geodesics. To avoid this problem, we could integrate over the surface for each $\lambda$ first and then combine them, giving

$$
A_{3}^{\prime}=\int d \lambda \int_{\Sigma(\lambda)} \sqrt{g_{2}} d \sigma_{1} d \sigma_{2} T_{a b} l^{a} l^{b} .
$$

However, Eq. (9), like Eq. (6), is not in an obvious way an average of ANEC.

We will not attempt to solve these problems, but rather we will exhibit counterexamples that apply to a very wide class of averaging procedures. We are able to do this because we work to first nonvanishing order in a spacetime that is a small perturbation of flat space. As we did in Ref. [10], we work in a conformally flat spacetime with conformal factor $\Omega=e^{\omega} \sim 1+\omega$, with $\omega \ll 1$. We define our average by letting $l^{a}$ be constant in the unperturbed spacetime and find violations of averaged versions of ANEC at order $\omega^{2}$. Suppose now that we use a different procedure. If we defined $l^{a}$ by parallel transport along a path which winds many times in the region where $\omega$ is largest, we could of course accumulate a large change in $l^{a}$. But this procedure is obviously pathological. If we restrict ourselves to a path which is free of such windings, the change in $l^{a}$ along a path $C$ will be given schematically by

$$
\Delta l^{a} \sim \int_{C} \Gamma_{b c}^{a} l^{b} d C^{c}
$$


If the scale of the curved region is given by $r$, the magnitude of $\Gamma_{b c}^{a}$ is of order $\omega / r$, so $\Delta l^{a} \sim \omega$. Thus the effect of the choice of path is of higher order in $\omega$ than the original effect and can be consistently neglected.

\section{CURVATURE ANOMALY}

We will generate violations using the anomalous curvature term in the conformal transformation of $T_{a b}$. First we will review the curvature anomaly for a general conformally flat space, before specifying a transformation. A conformally coupled field transforms as $\bar{\phi}=\Omega^{-1} \phi$, but the stress tensor has extra terms, given fully in [11]. Our analysis follows [10]. When beginning with Minkowski space, the Weyl tensor always vanishes. Terms proportional to $g_{a b}$ vanish upon null projection, while those proportional to $R_{; a b}$ vanish on integration along each geodesic. Considering only the remaining terms, we have

$$
\bar{T}_{a b}=\Omega^{-2} T_{a b}+2 \beta\left[\bar{R}_{a}^{c} \bar{R}_{c b}-\bar{R} \bar{R}_{a b}\right],
$$

where $\beta$ is given by Eq. (5).

Next we express these curvature quantities in terms of the conformal transformation, with $\omega=\ln \Omega$. Again dropping terms with $g_{a b}$, the stress tensor is given by

$$
\begin{array}{r}
\bar{T}_{a b}=\Omega^{-2} T_{a b}+8 \beta \Omega^{-2}\left[\omega^{, c}{ }_{a} \omega_{, c b}-2(\square \omega\right. \\
\left.+\omega^{, c} \omega_{, c}\right)\left(\omega_{, a b}-\omega_{, a} \omega_{, b}\right) \\
\left.-\omega^{, c} \omega_{, a} \omega_{, c b}-\omega^{, c} \omega_{, b} \omega_{, c a}\right]
\end{array}
$$

We take an initial state with $T_{a b}=0$, so the state does not contribute to $\bar{T}_{a b}$. We also have $\omega$ much less than one, so we may ignore terms of order $\omega^{3}$ and take $\Omega \approx 1$. That leaves us with only

$$
\bar{T}_{v v}=8 \beta\left[g^{c d} \omega_{, c v} \omega_{, d v}-2 \square \omega \omega_{, v v}\right]
$$

In our coordinates we organize this as

$$
\bar{T}_{v v}=8 \beta\left[\omega_{, x v}^{2}+\omega_{, y v}^{2}-2\left(\omega_{, u v}+\omega_{, x x}+\omega_{, y y}\right) \omega_{, v v}\right]
$$

We first study a particular transformation which gives a simpler violation of ANEC than (3), which is

$$
\omega=a x r^{-1} e^{-\rho},
$$

where we define $\rho=\left(u^{2}+v^{2}+x^{2}+y^{2}\right) / r^{2}$. This gives a localized transformation, so our spacetime is both conformally and asymptotically flat. The stress tensor component at $x=y=0$ is

$$
\bar{T}_{v v}=\frac{32 a^{2} v^{2} \beta}{r^{6}} e^{-2 \rho}
$$

Because $\beta$ is a negative number, this is always negative. Thus integrating over $\gamma$ for any fixed $u$ violates ANEC, so of course $A_{2}$ is negative as well. This gives a violation of greater magnitude as $a$ grows, but this analysis depends on $a \ll 1$ so it is not possible to build an arbitrarily large violation.

For averaging transversely and over the whole spacetime the above example does not give a negative answer, so instead we use

$$
\omega=(b u+c v) r^{-1} e^{-\rho}
$$


Any term which is odd in $x, y$, or $z$ will vanish on integration, so we do not write such terms. Including only the even terms, the $v v$ stress tensor component becomes

$$
\begin{aligned}
\bar{T}_{v v}= & \frac{32 \beta}{r^{10}}\left\{4 b^{2} u^{2}\left[\left(x^{2}+y^{2}\right)\left(r^{2}-v^{2}\right)+r^{2}\left(2 v^{2}-r^{2}\right)\right]\right. \\
& -2 b c\left[8 u^{2} v^{4}-2 r^{2}\left(v^{4}+5 u^{2} v^{2}\right)+r^{4}\left(u^{2}+3 v^{2}\right)\right] \\
& \left.+c^{2}\left[\left(x^{2}+y^{2}\right)\left(-4 v^{4}+8 v^{2} r^{2}+r^{4}\right)+4 r^{2}\left(2 v^{4}-3 r^{2} v^{2}\right)\right]\right\} e^{-2 \rho}
\end{aligned}
$$

To calculate $A_{3}$ (which coincides with $A_{3}^{\prime}$ to first order) we set $u=0$, and this becomes

$\bar{T}_{v v}=\frac{32 \beta}{r^{10}}\left\{2 b c\left(2 v^{4} r^{2}-3 v^{2} r^{4}\right)+c^{2}\left[\left(x^{2}+y^{2}\right)\left(-4 v^{4}+8 v^{2} r^{2}+r^{4}\right)+4 r^{2}\left(2 v^{4}-3 r^{2} v^{2}\right)\right]\right\} e^{-2 \rho}$.

Note that here, the $b^{2}$ term drops completely, but the $c^{2}$ term is entirely unchanged. The integral is

$$
A_{3}=-\frac{3 \sqrt{2} \pi^{3 / 2} \beta}{r} c(2 b+c)
$$

So long as $b<-c / 2$ this will be a negative quantity.

If instead we average (18) over the whole manifold, as in (6), we have

$$
A_{4}=-\beta \pi^{2}\left(b^{2}+6 b c+3 c^{2}\right)
$$

For $-3-\sqrt{6}<b / c<-3+\sqrt{6}$, the average is negative. As discussed earlier, these results still hold even if the integrand differs by any power of $\Omega$. Here all dependence on $r$ has dropped, and thus the sharpness of the curvature does not affect the violation.

\section{DISCUSSION}

We have demonstrated counterexamples for generally averaged null energy conditions, with averaging over a timelike 2-surface, a null 3-surface, and the entire manifold. We now find it unlikely there is any principle that would rule out exotic phenomena by restricting the total set of possible stress-energy tensor configurations without regard to the background. If so, we must restrict our attention to self-consistent fields, that is, quantum fields that give rise to an exotic spacetime with that same spacetime, rather than an arbitrary different one, as the background. In other words, the entire system should satisfy the semiclassical Einstein equation,

$$
G_{a b}=8 \pi G\left(\left\langle T_{a b}^{\text {quantum }}\right\rangle+T_{a b}^{\text {classical }}\right) .
$$

Here $T^{\text {classical }}$ is a classical stress tensor that obeys energy conditions, and $T^{\text {quantum }}$ is the stress tensor of some state of a set of quantum fields, all in the background whose Einstein tensor is $G_{a b}$.

In the previous sections, we found violations of ANEC and its averages in small perturbations around Minkowski space. The same situation was studied for self-consistent systems by Flanagan and Wald [14], who found that ANEC was obeyed, at least for pure states. In fact, no violations of "self-consistent achronal ANEC" are known, and it is powerful enough to rule out many problematic spacetimes [5]. Nevertheless, it could be the case that even this 
condition needs to be weakened by additional averaging. It is also possible that a negative yet finite state-independent lower bound gives a useful condition, as discussed in Ref. [15]. The first example considered in Ref. [10] violates singly averaged ANEC without bound, but with additional averaging the examples considered here are all finite. Unfortunately, it is difficult to define such an average outside of perturbation theory, as discussed in Sec. II.

\section{Acknowledgments}

The authors thank Chris Fewster and Tom Roman for helpful discussions. This research was supported in part by Grants No. RFP1-06-024 and No. RFP2-06-23 from The Foundational Questions Institute (fqxi.org).

[1] J. L. Friedman, K. Schleich, and D. M. Witt, Phys. Rev. Lett. 71, 1486 (1993), gr-qc/9305017.

[2] K. D. Olum, Phys. Rev. Lett. 81, 3567 (1998), gr-qc/9805003.

[3] F. J. Tipler, Phys. Rev. Lett. 37, 879 (1976).

[4] S. W. Hawking, Phys. Rev. D46, 603 (1992).

[5] N. Graham and K. D. Olum, Phys. Rev. D76, 064001 (2007), 0705.3193.

[6] G. Klinkhammer, Phys. Rev. D43, 2542 (1991).

[7] R. M. Wald and U. Yurtsever, Phys. Rev. D44, 403 (1991).

[8] C. J. Fewster, K. D. Olum, and M. J. Pfenning, Phys. Rev. D75, 025007 (2007), grqc/0609007.

[9] M. Visser, Phys. Lett. B349, 443 (1995), gr-qc/9409043.

[10] D. Urban and K. D. Olum (2009), 0910.5925.

[11] D. N. Page, Phys. Rev. D25, 1499 (1982).

[12] C. J. Fewster and T. A. Roman, Phys. Rev. D67, 044003 (2003), erratum: D80, 069903(E).

[13] C. W. Misner, K. S. Thorne and J. A. Wheeler, Gravitation (W. H. Freeman and Company, 1973).

[14] E. E. Flanagan and R. M. Wald, Phys. Rev. D54, 6233 (1996), gr-qc/9602052.

[15] U. Yurtsever, Phys. Rev. D52, R564 (1995), gr-qc/9501040. 